\title{
Programas parentales eficaces para el tratamiento de la conducta disruptiva: El caso del programa Empoderando Padres, Empoderando Comunidades
} Cost-effective Parental Programs for the Treatment
of Disruptive Behavior: The case of the Empowering
Parents, Empowering Communities program

William James Center for Research, ISPA-Instituto Universitłrio, Lisboa. Portugal Miguel Basto-Pereira

William James Center for Research, ISPA-Instituto Universittrio, Lisboa. Portugal Crispin Day

Department of Psychology, Child \& Adolescent Mental Health Service Research Unit, King's College London, Institute of Psychiatry, Psyhcology and Neuroscience, and Centre for Parent and Child Support, South London and Maudsley NHS Foundation Trust, Michael Rutter Centre, Londres.

\section{resumen/abstract:}

Los programas parentales tempranos son conocidos como una de las estrategias más eficaces para aliviar algunas de las consecuencias más negativas de los problemas conductuales infantiles. A pesar de que se han creado diversas intervenciones familiares, todavía existen limitaciones que necesitan superarse. Empoderando Padres, Empoderando Comunidades (EPEC) es un programa parental fundado en la evidencia, con el objetivo principal de promover el desarrollo infantil saludable, al mismo tiempo que pretende reforzar las habilidades parentales, el funcionamiento familiar y el bienestar parental. Asentada en las teorías del apego, sistemas familiares, y teoría cognitivo-conductual, su enfoque único liderado por iguales ha arrojado beneficios, no solo en el bienestar de los niños y sus familias, sino también en el de líderes de grupos de padres e iguales, y comunidad en general. En la actualidad, EPEC se ha puesto en marcha en Reino Unido y Australia, mostrando altos niveles de aceptabilidad y eficacia. También está previsto que se aplique en otros países no anglo-sajones. Por lo tanto, el objetivo de este trabajo es el de revisar la evidencia en torno al programa EPEC, y analizar sus ventajas, limitaciones, y líneas futuras. Asimismo, también se debate sobre la importancia de su difusión a nivel mundial.

Early parenting programs are known to be one of the most effective strategies to alleviate some of the negative impacts of childhood behavior problems. Although several family interventions have been created, there were still limitations that needed to be addressed. Empowering Parents, Empowering Communities (EPEC) is an evidence-based, cost-effective parenting program with the main purpose of promoting healthy childhood development, while also strengthening parenting skills, family functioning and parental wellbeing. Based on attachment, family systems and cognitive-behavioral theories, its unique peer-led approach has shown immediate benefits not only to the wellbeing of children and their families, but also to peer parent group leaders and the community in general. EPEC is presently implemented in the UK and Australia, showing high levels of acceptability and effectiveness. It is also set out to be applied in other non-Anglo-Saxon countries. Therefore, the aim of this paper is to review the evidence regarding the EPEC program and analyze its advantages, limitations, and future directions. The importance of its dissemination worldwide is also discussed.

\section{palabras clave/keywords:}

Programa parental; Infancia; Problemas conductuales; Parentalidad positiva

Parenting program; Childhood; Behavior problems; Positive parenting 


\section{Introducción}

A nivel mundial, se estima que la prevalencia de problemas de salud mental infantil es de un $20 \%$, siendo los problemas de conducta una de las psicopatologías más frecuentes (Vasileva et al., 2020). Esto incluye una amplia gama de comportamientos disruptivos (por ejemplo, impulsividad, agresión), que se convierten en clínicamente significativas cuando son persistentes e incapacitantes. Diversos estudios (Bevilacqua et al., 2018; Jolliffe et al., 2017) han analizado el impacto negativo de la conducta disruptiva, como la agresión y la desobediencia, en la calidad de vida tanto de los niños como de sus familias. De hecho, las consecuencias más negativas son el desarrollo de trayectorias evolutivas problemáticas como el fracaso académico, la delincuencia juvenil y la marginación social posterior, con los consecuentes costes sociales y económicos.

Este problema ha supuesto una preocupación para la Organización Mundial de la Salud (OMS, 2016), que ha desarrollado estrategias para reducir el impacto y promover el desarrollo. En este contexto, los programas parentales son conocidos por constituir una de las estrategias más efectivas para aliviar algunos de los efectos negativos de los problemas infantiles (Kaminski y Claussen, 2017). Los resultados de estos programas han arrojado beneficios inmediatos al reducir el riesgo de conducta antisocial futura y de carreras delincuenciales de inicio temprano (Farrington et al., 2017), promocionando vías de desarrollo más positivas (Pedersen et al., 2019) y mejorando el funcionamiento familiar (Barlow y Coren, 2017).

Además, de acuerdo con lo anterior, las intervenciones tempranas son más eficaces que las intervenciones posteriores en la vida del individuo. Esto se debe a que el desarrollo infantil puede ser más maleable durante los primeros años de vida, cuando el cerebro en desarrollo es más responsivo a las influencias ambientales (Wachs et al., 2014). Como consecuencia, se han llevado a cabo esfuerzos internacionales para desarrollar y evaluar programas parentales tempranos y proporcionar a las familias entrenamiento y habilidades que les ayuden a atender mejor las habilidades físicas, mentales y sociales de sus hijos.

Intervenciones como la Triple P (Sanders, 1999), Los Increíbles Años (Webster-Stratton, 2001) y la Terapia de Interacción Padre-Hijo (Eyberg et al., 1995) son algunos de los programas parentales más conocidos, con el objetivo principal de reducir las conductas disruptivas, mostrando una razonablemente buena efectividad. Sin embargo, la literatura sugiere que las familias que más necesitan el tratamiento a menudo lo abandonan de forma temprana o son incapaces de implicarse desde el inicio (Cortis, 2012). Parte de la dificultad para implicarse puede explicarse por cuestiones logísticas, como falta de tiempo o dificultades con el transporte. Igualmente, puede provenir de expectativas negativas de los padres sobre cursos parentales y preocupación sobre cómo se les percibe en un contexto clínico (Tully et al., 2017). Estos resultados sugieren que existe la necesidad de programas con altos niveles de aceptación, aprobación social y bajo estigma, que puedan prevenir simultáneamente conductas parentales inadecuadas y que apoyen emocionalmente a los padres.

En este contexto, el Programa Empoderando Padres, Empoderando Comunidades (EPEC; Day et al., 2012) se creó con el propósito de aumentar la implicación de poblaciones de alto 
riesgo y de reducir las tasas de abandono en las intervenciones parentales. Es un portafolio de cursos parentales, liderado por iguales, con altas tasas de éxito y coste-beneficio, que mejora el bienestar social, emocional y conductual de los niños y de los padres. Su combinación única de intervención liderada por iguales y reforzada por supervisión profesional, garantía de calidad y entrenamiento, resulta innovadora en el ámbito de los problemas conductuales infantiles. De hecho, Piquero y colaboradores (2016) llevaron a cabo un meta-análisis para comprobar la eficacia de programas parentales tempranos y sus resultados mostraron que EPEC era el más efectivo al compararlo con entrenamientos parentales similares, no solo para reducir la conducta disruptiva, sino también para mejorar las habilidades parentales.

Por ello, proponemos en este trabajo revisar la literatura sobre el programa EPEC, los estudios existentes sobre su efectividad y difusión, así como analizar sus ventajas y limitaciones.

\section{Intervenciones EPEC}

\section{Ámbito, Objetivos y Estructura}

EPEC se creó en Gran Bretaña en 2012, gracias al Centro de Apoyo para Padres e Hijos, de la South London and Maudsley NHS Foundation Trust (SLAM), y la Child \& Adolescent Mental Health Services (CAMHS) Research Unit, del King's College London. Su variedad de intervenciones estaba diseñada con el objetivo principal de promocionar el desarrollo social, emocional, conductual y académico infantil, al tiempo que incrementaba las habilidades parentales, la confianza y el bienestar, y mantenía un funcionamiento familiar saludable (Day et al., 2020). Por lo tanto, EPEC proporciona sistemas asentados en la evidencia para ayudar a los padres a aprender habilidades parentales prácticas para la vida diaria familiar, y educar niños seguros, felices y cooperativos (ver Figura 1).

Para alcanzar estos objetivos, muchos psicólogos, líderes de grupos de padres, y padres resultaron esenciales para desarrollar y coproducir los cursos EPEC. De hecho, un rasgo importante de EPEC lo constituye el hecho de que fue creado con el intento de ser liderado por iguales, padres locales, que hubieran completado con éxito un curso de Entrenamiento Facilitador de Iguales EPEC. Además, muchos de los facilitadores de iguales fueron padres que previamente habían asistido a uno de los cursos incluidos en EPEC. Con el fin de asegurar sesiones eficaces, los padres facilitadores recibían supervisión y apoyo de forma regular por parte de los especialistas parentales ubicados en su centro EPEC (Day et al., 2020). Estos centros se localizaban en servicios locales donde las familias y comunidades presentaban una alta exposición a factores de riesgo asociados con resultados negativos infantiles y familiares, así como baja resiliencia (Day et al., 2020). La intención era que EPEC ayudara a incrementar el acceso y la magnitud del apoyo parental eficaz, reduciendo la estigmatización y el riesgo familiar.

En este contexto, existen tres cursos principales EPEC pautados para las diferentes etapas del desarrollo, en concreto: a) "Ser Padre", para padres de niños entre los 2-11 años; b) "El Bebé y Nosotros", para padres de bebés entre 0-1 años, y c) "Viviendo con Adolescentes", para padres de adolescentes entre 12-16 años (Day et al., 2020). Además, existe una gama 
de cursos EPEC más focalizados, para padres de niños con Trastorno por Déficit de Atención e Hiperactividad (TDAH), padres de niños con Trastorno del Espectro Autista (TEA), parejas co-parentales con riesgo de conflicto, familias viviendo en alojamientos temporales, y padres afectados por dificultades de salud mental graves (Day et al., 2020).

Los cursos parentales por lo general consisten en sesiones de 2 horas facilitadas por dos líderes locales de grupos parentales acreditados, para 8-12 padres. Fundada en las teorías del apego, sistemas familiares y enfoques cognitivo-conductuales, estas sesiones integran con éxito adquisición de conocimiento, cambio conductual, con aprendizaje adulto y apoyo social. Cada sesión del curso es muy interactiva, implicando una mezcla interesante y creativa de debate en pequeño y gran grupo, representaciones de situaciones, ejemplos, e información compartida y reflexión sobre la misma. Esto capacita a los padres a practicar y

Figura 1.- Funcionamiento del programa Empoderando Padres, Empoderando Comunidades (Elaboración propria)

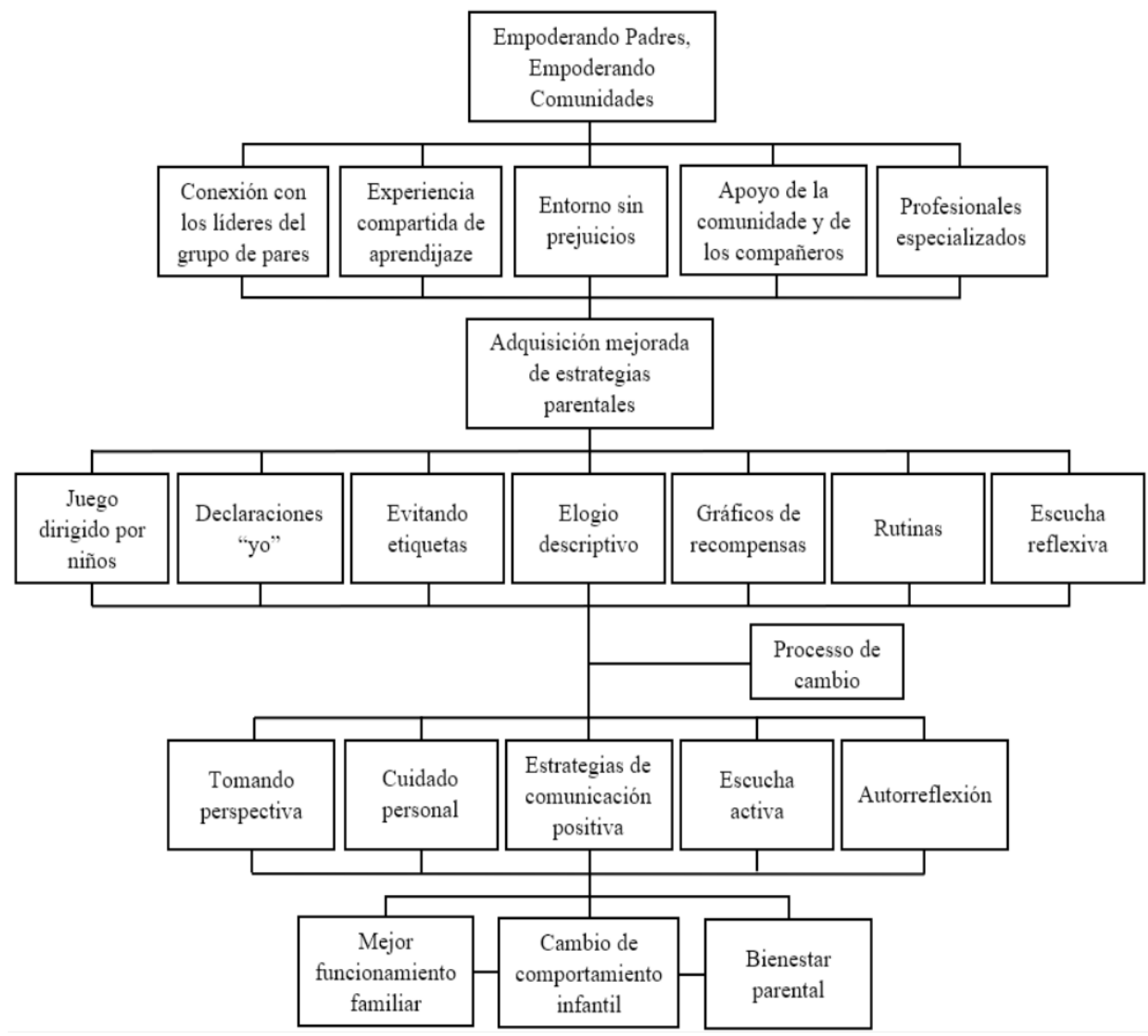


utilizar nuevas habilidades para conseguir objetivos específicos (Day et al., 2020).

La Tabla 1 muestra el guía del curso "Ser Padre". Este curso es el más importante del programa EPEC, y es la base para todos los demás cursos del programa EPEC. De esta manera, este curso, así como los demás cursos pertenecientes a EPEC, combina la ciencia y teoría evolutiva más reciente con las estrategias parentales basadas en la evidencia que se centran en las problemáticas presentes habitualmente en los problemas conductuales infantiles (Day et al., 2020). De hecho, numerosos ensayos clínicos aleatorizados muestran que las intervenciones parentales que mejoran estas dimensiones pueden reducir la conducta negativa infantil (por ejemplo, Leijten et al., 2019; Meija et al., 2017). Para obtener más información sobre el funcionamiento de EPEC, puede consultarse el Anexo (Day y Nicoll, 2017).

Tabla 1.- Guía del curso 'Ser Padre'

\begin{tabular}{ll}
\hline Sesión 1: Ser Padre & Sesión 2: Sentimientos \\
\hline 'Bastante bueno' vs padre 'perfecto' & Reconociendo y aceptando sentimientos \\
Cuidando de nosotros mismos & Expresando sentimientos \\
\hline Sesión 3: Juego & Sesión 4: Valorando a Mi Hijo \\
\hline Juego dirigido por niños & Evitando 'etiquetas' \\
& Utilizando la alabanza \\
\hline Sesión 5: Comprendiendo la conducta & Sesión 6: Estrategias de Disciplina \\
\hline Necesidades del niño & Límites \\
Disciplina & Tiempo fuera y decir 'No' \\
Órdenes, consecuencias y mapas celestes & Reglas del hogar \\
\hline Sesión 7: Escucha & Sesión 8: Revisión y Apoyo \\
\hline Estilos comunicativos & Afrontando el estrés \\
Escucha reflexiva & Revisando \\
Preguntas 'abiertas' y 'cerradas' & Sabiendo dónde conseguir apoyo \\
\hline
\end{tabular}

Nota. Adaptado de Ser Padre: Manual para Facilitadores de Curso (4 ${ }^{\text {th }}$ ed, p. 12) por C. Penney, C. Wilson, L. Draper, C. Day y C. Kearney, 2018. Copyright 2018 de Caroline Penney, Charlotte Wilson, Lucy Draper, Crispin Day y Catherine Kearney

\section{Antecedentes teóricos}

Los principales conceptos teóricos tras el desarrollo de los cursos EPEC son la teoría del aprendizaje social, el apego, y los enfoques basados en las relaciones (Day et al., 2020). Ciertamente, numerosos ensayos clínicos (por ejemplo, Baker et al., 2017) han demostrado que los programas parentales desarrollados a partir de las teorías del aprendizaje social y del apego, mejoran la conducta disruptiva en los niños.

La teoría del aprendizaje social propone que las experiencias reales del niño y las exposiciones moldean la conducta, directa o indirectamente (Scott y Gardner, 2015). Por lo tanto, 
las estrategias infantiles para manejar emociones, resolver conflictos e implicarse con otros se aprenden de la experiencia y se generalizan a través de los contextos y del tiempo (Akers y Jennings, 2019). Especialmente en niños más pequeños, la fuente primaria de estas experiencias la constituye el ambiente relacional familiar y padre-hijo.

Por otra parte, la teoría del apego propone que la calidad del cuidado proporcionado al menor, especialmente la sensibilidad y la responsividad, conducen a un apego seguro o inseguro. En esta línea, los teóricos han encontrado que una forma concreta de apego no seguro en bebés y niños pequeños está estrechamente asociada al riesgo de psicopatología (Scott y Gardner, 2015). Esto es debido al hecho de que las relaciones de apego se internalizan y se generalizan para influir en las expectativas de otras relaciones importantes (Scott y Gardner, 2015). Por lo tanto, una historia de inconsistencia y cuidado no sensible por parte de un cuidador principal se espera que conduzca al niño a desarrollar un modelo disfuncional de sí mismo y de los demás.

Existen muchas otras teorías parentales, algunas con una considerable base de evidencias. Una de las que ha influido en las intervenciones parentales, como en EPEC, es el enfoque de estilos parentales. Se basa en dimensiones centrales de la parentalidad (por ejemplo, autoritativo, autoritario, permisivo y no implicado/negligente) que pueden afectar al desarrollo infantil (Scott y Gardner, 2015). En este contexto, los niños cuyos padres son descritos como autoritarios, permisivos o no implicados, muestran los resultados significativamente más negativos. Trabajar con los padres para conseguir un estilo parental más autoritativo (por ejemplo, más control asertivo, alta calidez y altas expectativas en la adolescencia), es uno de los objetivos de EPEC. Se ha encontrado que los niños de padres autoritativos son consistentemente más prosociales, más competentes académica y socialmente, y menos sintomáticos (Majumder, 2015).

De hecho, el resultado de que la calidad de la relación padre-hijo esté asociada con problemas conductuales infantiles es uno de los hallazgos más ampliamente informados en la bibliografía (por ejemplo, estudios de Cambridge e Isle of Wight). De forma coherente, las intervenciones cuyo objetivo principal es mejorar la conducta infantil, como EPEC, se centran en modificar la calidad de las habilidades y estilos parentales, así como la relación padre-hijo.

Las intervenciones EPEC se apoyan asimismo en la teoría de sistemas familiares, un marco conceptual de trabajo esencial al tratar con familias. No en vano, los teóricos han encontrado que existe una interacción compleja adicional entre el efecto del ambiente familiar, y la personalidad y características temperamentales del niño (Eisler y Lask, 2015). Las familias necesitan proporcionar estabilidad y predictibilidad, pero también necesitan ser capaces de cambiar para adaptarse a nuevas circunstancias y a las demandas evolutivas de los miembros familiares a nivel individual (Eisler y Lask, 2015). Por ejemplo, la necesidad infantil de dependencia y apego requiere de un grado de estabilidad y constancia en la familia. Sin embargo, a medida que el niño crece, la familia también debe encontrar nuevas formas de satisfacer sus necesidades de independencia y separación. Cuando la familia evoluciona a través de las previsibles etapas del ciclo de vida familiar, necesita ser capaz de adaptarse 
y cambiar su estilo habitual de funcionamiento (Eisler y Lask, 2015). Estas etapas previsibles del ciclo de vida familiar pueden complicarse por acontecimientos inesperados (por ejemplo, enfermedad, muerte, separación familiar), y el estrés combinado puede contribuir al desarrollo de psicopatología. La manera en que la familia responde a este problema emergente es un factor crucial para determinar el alcance de la vulnerabilidad individual a estos desafíos (Eisler y Lask, 2015).

Si se analiza más de cerca el formato de distribución de EPEC, sus cursos parentales se aplican a grupos de padres, con un formato interactivo y de aprendizaje colaborativo que incluye elementos clave como la observación, las representaciones de situaciones y los debates grupales. Según la evidencia empírica, no solo las intervenciones grupales son mejores en coste-beneficio (Nystrand et al., 2019), sino que también parecen disminuir los sentimientos de aislamiento de los padres (Tully et al., 2017). Cuando los padres desarrollan relaciones con familias que presentan problemas similares, parece reducirse el estigma de contar con un hijo problemático al tiempo que mejora la percepción que las familias tienen sobre el apoyo social (Tully et al., 2018). Los tratamientos en grupo también presentan oportunidades de crear una cultura de presión positiva por parte de los iguales entre las familias, a la vez que generan esperanza y motivación, lo cual puede jugar un papel importante a la hora de implicar a los padres en el tratamiento y en la asistencia creciente (Yalom y Leszcz. 2020).

En paralelo, el programa EPEC originalmente se desarrolló también utilizando un enfoque liderado por los iguales. Esto se debe a que se hallaron importantes beneficios en los modelos de salud mental distribuidos por iguales. Por ejemplo, una revisión de Hoagwood y colaboradores (2010) encontró que aunque los resultados fueron mixtos, los programas distribuidos por iguales o coliderados por un igual y un profesional de la salud mental enriquecían el conocimiento sobre la salud mental del cuidador, su iniciación en los servicios de salud mental infantil, su empoderamiento y su satisfacción. Posteriormente, y en respuesta a la falta de diseños de investigación rigurosos utilizados para evaluar la mayoría de estos programas, Acri y colaboradores (2017) llevaron a cabo una revisión de estudios que utilizaba ensayos controlados aleatorizados. Los resultados fueron similares a los de la revisión de Hoagwood y sus colaboradores, ya que se encontraron mejoras significativas en el funcionamiento familiar, conocimiento sobre enfermedad mental y enriquecimiento de las habilidades parentales en los seis programas distribuidos por iguales incluidos en el estudio (Acri et al., 2017).

A pesar del tremendo crecimiento de los enfoques basados en iguales, y aunque el modelo ha existido durante más de dos décadas, parece existir un aparente vacío en la distribución de los programas de entrenamiento parental, lo cual es especialmente destacable dado que la demanda de programas de habilidades parentales es alta, y las dificultades conductuales internalizantes se encuentran entre las razones más comunes para derivar a los servicios de salud mental (Acri et al., 2017). De hecho, el uso del enfoque liderado por iguales es una de las ventajas del programa EPEC. Estos cursos de entrenamiento parental incluyen una combinación innovadora de métodos liderados por iguales junto al apoyo y supervisión parental profesional. 


\section{Eficacia en Gran Bretaña y más allá}

El primer estudio piloto sobre EPEC (Day et al., 2012a) era un diseño de cohorte pre-post llevado a cabo en Gran Bretaña y que incluía 31 facilitadores entrenados de iguales y 73 participantes que asistían al curso "Ser Padre". Los padres informaron de mejoras en el comportamiento infantil y en el estrés parental, así como de una alta satisfacción. Asimismo, los facilitadores mostraron un aumento significativo de la confianza y del conocimiento a la hora de tratar con grupos de padres.

Posteriormente, se llevó a cabo un ensayo controlado aleatorizado, basado en la comunidad, para evaluar la eficacia del curso "Ser Padre", de niños de 2-11 años de edad (Day et al., 2012b). Los resultados mostraron que este curso parental específico mejoró de forma extraordinaria los resultados para la conducta disruptiva infantil $(d=.38, \mathrm{p}=.01)$, la conducta parental positiva $(d=.69, \mathrm{p}<.001)$, al tiempo que disminuyeron las preocupaciones parentales $(d=.77, \mathrm{p}<.001)$. De hecho, esta evidencia sugiere que EPEC podría ser un modelo de servicio eficaz y aceptable en comunidades con desventaja social.

Desde entonces, y con la creación tanto de cursos paralelos (por ejemplo, "El Bebé y Nosotros"), como de cursos más específicos ("Ser Padres Juntos"), EPEC ha sido evaluado rigurosamente en ensayos de investigación y ha sido probado en el mundo real. Existen nueve estudios publicados de EPEC que muestran que los cursos parentales mejoran el desarrollo social, emocional y conductual infantil, el bienestar parental, la confianza, la resiliencia (Day et al., 2020) e incrementan la función reflexiva parental (Harwood, 2017). De forma adicional, el Programa Scalling EPEC (Day et al., 2020) corrobora estos resultados y prueba que es posible reproducir los resultados cuando la intervención es realizada por equipos independientes.

Es más, la evidencia muestra que el Entrenamiento para Facilitador de Iguales es eficaz e incrementa de forma significativa el conocimiento parental y las habilidades de facilitación, al tiempo que más del $90 \%$ de los padres que han completado EPEC califican muy alto a sus líderes grupales en términos de competencia, conocimiento y habilidades motivacionales (Day et al., 2020; Day et al., 2017). En la misma línea, un estudio cualitativo de Thomson y colaboradores (2014) mostró que la experiencia de ayudar a los padres a conseguir resultados positivos es emocionalmente reforzante para los facilitadores de iguales, provocando efectos positivos recíprocos en la motivación y autoeficacia de los facilitadores. Esto ayuda a mantener una alta fidelidad para seguir los procedimientos según los protocolos y para el compromiso a largo plazo. Las ganancias personales, como la mejora del estatus social y de las relaciones familiares también refuerzan la participación de los facilitadores.

EPEC también ha sido valorado independientemente como un programa parental eficaz, y de bajo coste, por organizaciones independientes en Gran Bretaña y Australia (Early Intervention Foundation, EIF, 2016; Australian Institute of Family Studies, AIF, 2015), demostrando de forma consistente que no solo puede mejorar el bienestar infantil y parental, sino también generar altos niveles de implicación parental y experiencias positivas, así como conectividad social y de comunidad entre las familias (Day et al., 2020). 
Asimismo, EPEC presenta las tasas más bajas de coste, y de comparación de coste, según Early Intervention Foundation (EIF, 2016). En comparaciones realizadas con costes estimados publicados por el National Institute of Health and Care Excellent de Gran Bretaña (NICE, 2013), EPEC puede reducir potencialmente los costes al 75\%, comparado con otros programas parentales establecidos y liderados por profesionales.

Siguiendo los prometedores resultados obtenidos en Gran Bretaña, el curso "Ser Padre" de EPEC se pilotó y evaluó en Tasmania (Australia), entre 2011 y 2014. Manteniendo los mismos principios del programa original británico, los cursos "Ser Padre" se llevaron a cabo en los Child and Family Centers (CFC), y cuando estas instalaciones no estaban disponibles, las iglesias y colegios fueron la siguiente opción. La evaluación de Tasmania no solo halló altas tasas de permanencia en el curso (79\%), sino que también los padres y facilitadores hablaron de "incrementos en el capital social, humano y de identidad, mientras que las comunidades implicadas en EPEC demostraron un aumento de la capacidad social en torno a las habilidades parentales" (Winter, 2013, p. 38). Los padres también informaron de una mejor escucha, más entendimiento y conocimiento de sus habilidades parentales, una visión más optimista de la vida, y una mejora de las relaciones padre-hijo. Asimismo, los padres que continuaron al siguiente nivel EPEC y obtuvieron el Entrenamiento de Facilitador de Iguales informaron de un aumento de la confianza, optimismo, vías de desarrollo educacional y de empleo, y una mejora de la habilidad para manejar los desafíos de la vida diaria (Winter, 2013).

El curso EPEC para padres con Trastorno del Espectro Autista (TEA) está actualmente en proceso de adaptación en Ningbo (China). El hecho de adaptar una intervención parental anglo-sajona a un contexto no anglo-sajón puede ser una tarea complicada y que requiere mucho esfuerzo, dado que se necesitan una serie de modificaciones culturales para completar una adaptación eficaz. Para conseguir este objetivo, los autores seguirán el modelo compilado de adaptación de intervenciones, que comprende el Modelo Validez Ecológica (Bernal et al., 1995, 2009), y el Proceso de Adaptación Cultural (Domenech-Rodríguez y Wieling, 2004).

El curso "Ser Padre" también está planteado para pilotarse en Portugal en 2022. El número de casos relacionados con conducta disruptiva juvenil informados en Portugal se ha ido incrementando de forma consistente, y a pesar de que se realiza un seguimiento a los padres con hijos con problemas de conducta, por parte de los servicios locales portugueses de protección de menores, no existen respuestas implementadas basadas en la evidencia en este contexto. Esto es debido al hecho de que no muchos programas parentales centrados en los problemas conductuales infantiles, se han adaptado al contexto portugués, y los que lo han hecho (por ejemplo, Años Increíbles), no presentan una buena ratio coste-beneficio. Por lo tanto, es más que pertinente evaluar la eficacia de EPEC, más específicamente del curso "Ser Padre", en los servicios locales portugueses de protección de menores.

Se realizarán los cambios culturales y de distribución pertinentes, siguiendo un modelo compilado de adaptación de intervenciones, que comprende el Modelo de Validez Ecológica (Bernal et al., 1995, 2009), y el Proceso de Adaptación Cultural (Domenech-Rodríguez y 
Wieling, 2004). El principal cambio sería que, en lugar de utilizar un enfoque liderado por los iguales, la versión portuguesa de "Ser Padre" se llevará a cabo por profesionales del Sistema de Protección de Menores. Este cambio específico se debe al hecho de que los padres de niños con problemas de conducta siempre son derivados al sistema de protección. Este trabajo puede proporcionar a los profesionales de este sistema, dependiendo de los resultados del ensayo, una herramienta esencial y rentable para tratar los problemas de estos niños.

Esta adaptación del curso "Ser Padre" al contexto portugués es de máxima importancia. Estudiar la aceptación y eficacia de EPEC será un paso importante para analizar la solidez universal de este programa, ya que será implementado en un país con normas y valores diferentes sobre la parentalidad, así como prácticas familiares, factores políticos y religiosos, y también contextos de servicio distintos. De hecho, pretende ser el primer ensayo controlado aleatorizado de EPEC fuera de Gran Bretaña, lo que le da un enorme valor a su diseminación.

\section{Ventajas y limitaciones}

Se han asociado muchos beneficios a los cursos EPEC. En primer lugar, las teorías que apoyan este programa (por ejemplo, el aprendizaje social y los enfoques basados en las relaciones) y sus componentes (refuerzo positivo, escucha reflexiva, comunicación mejorada, incremento de la función parental reflexiva y relaciones cálidas padre hijo), han sido reconocidos como los métodos más eficaces para prevenir y tratar la conducta disruptiva, promover la parentalidad positiva y el funcionamiento familiar (por ejemplo, Leijten et al., 2019).

Asimismo, el enfoque liderado por iguales ayuda a los padres a construir relaciones de confianza con los iguales, haciendo que se sientan motivados y bien informados. Este tipo de enfoque también conlleva numerosos beneficios para los facilitadores de iguales, ya que mejora la confianza de los líderes, sus cualificaciones, y su empleabilidad, y ofrece a los líderes grupales una manera de expresar su altruismo personal y comunitario.

Los cursos EPEC por lo general constan de ocho sesiones (Day et al., 2020). Esto es en realidad un beneficio dado que es una intervención relativamente breve. Y esto es especialmente importante, ya que la evidencia empírica (por ejemplo, O'Brien y Daley, 2011) ha demostrado que las intervenciones parentales extensas son, no solo un desafío para que los padres asistan en términos de organizar el cuidado infantil, el transporte y las prioridades familiares que compiten entre sí, sino que también implican muchos recursos, son costosas y requieren un tiempo clínico muy significativo a través del entrenamiento y la supervisión.

Otra ventaja asociada a EPEC es que comprende una gama de cursos según protocolo que puede aplicarse a muestras específicas de niños y padres (por ejemplo, niños con TEA, padres sin hogar), en lugar de desarrollar un único curso que pueda "ajustarse a todos". Además, dado que está implementado en Gran Bretaña desde 2012, tiene a su disposición múltiples centros EPEC y una gran red de especialistas, facilitadores parentales y padres que juntos hacen posible el desarrollo continuo y la aplicación y mejora de sus cursos. De hecho, es su composición única y su implementación eficaz la que favorece que EPEC presente altas tasas de permanencia y mantenga un bajo coste. 
Además, la investigación muestra que EPEC: a) es uno de los programas parentales de Gran Bretaña con más evidencias; b) implica con éxito a padres en desventaja social y comunidades excluidas; c) transforma las colaboraciones entre servicios-comunidad y moviliza los recursos de la comunidad; d) crece rápidamente, multiplicando el alcance de los servicios sin socavar el suministro; y e) es muy popular y altamente valorado por una amplia gama de padres (Day et al., 2020). En resumen, EPEC está respaldado por una rigurosa investigación y evidencia práctica que muestra un impacto significativo en los resultados sociales, emocionales y conductuales infantiles, en la parentalidad, en la resiliencia familiar y en el capital social.

A pesar de lo anterior, el programa EPEC, como otras intervenciones parentales, también presenta sus limitaciones. La mayor de ellas es que es un enfoque de una sola faceta, es decir, que únicamente está centrado en la parentalidad. Y aunque las habilidades y prácticas parentales se encuentran asociadas a la conducta disruptiva infantil y al desarrollo infantil en general, necesitarían considerarse y trabajarse también otros factores, como los rasgos infantiles y la influencia de otras figuras de apoyo. Por lo tanto, es más probable que un enfoque con múltiples facetas, que incluyera no solo los cursos parentales, sino también intervenciones sobre los niños y los profesores, fuera más eficaz (Webster-Stratton y Reid, 2017).

Además, al mismo tiempo que EPEC muestra evidencia sobre la eficacia de la intervención parental liderada por iguales en los problemas conductuales infantiles, es importante reconocer algunos de los potenciales desafíos de utilizar el formato liderado por iguales. Los facilitadores de iguales pueden demandar altos niveles de supervisión regular y de alta calidad, así como una guía para asegurar un adecuado conocimiento y adquisición de habilidades al término del entrenamiento. Existe también la posibilidad de que algunos facilitadores de iguales puedan necesitar un período de tiempo más extenso para completar los portafolios del entrenamiento, o incluso que se desvinculen de llevar a los grupos (Day et al., 2012b). En este contexto, sería interesante evaluar la eficacia de los cursos EPEC liderados por profesionales, en lugar de por iguales, y analizar y comparar los resultados entre ambos métodos.

Por último, no existe evidencia directa de la eficacia a largo plazo EPEC. Aunque sus resultados son consistentes con otros programas parentales que informan sobre la eficacia a largo plazo en los problemas de conducta infantil, no puede concluirse que EPEC presente estos efectos duraderos. Sería necesaria la realización de estudios longitudinales para evaluar los efectos a largo plazo de los cursos EPEC en el bienestar infantil y de los progenitores.

\section{Conclusiones}

Se ha debatido en gran medida la importancia de desarrollar intervenciones con una buena relación coste-beneficio para prevenir la aparición de trayectorias infantiles anómalas. De hecho, incluso en los países desarrollados, una gran cantidad de niños presenta una salud física y emocional muy deficiente, y por lo tanto, se ha dedicado una atención creciente a la influencia de las intervenciones tempranas en el desarrollo psicológico y social infantil. Todas las conclusiones apuntan a que los programas parentales son una opción eficaz y valiosa 
para disminuir los problemas conductuales infantiles, así como para aumentar la probabilidad de un desarrollo normalizado y mejorar la función parental y familiar.

Los cursos EPEC contribuyen a esta evidencia, y de forma adicional, aportan un nuevo enfoque a la bibliografía existente sobre la conducta disruptiva. Sin lugar a dudas, EPEC no es solo una intervención más, facilitada por profesionales. De hecho, es el primer programa parental para problemas de conducta infantil facilitado por padres.

EPEC es un modelo que desafía los constructos de servicio jerárquico tradicional y allana el camino para permitir a los miembros de la comunidad trabajar junto a los profesionales.

Aunque todavía queda mucho por hacer, la investigación sobre EPEC ha arrojado resultados satisfactorios en Gran Bretaña y Australia. Estos resultados, y el hecho de que los cursos continúen desarrollándose actualmente en estos países, incita a pensar que EPEC puede adaptarse a otros países. Si se encontraran resultados similares, podría convertirse en una estrategia universal y asequible para ser implementada ante problemas infantiles, previniendo por tanto tasas de delincuencia y criminalidad alrededor del mundo.

Financiamiento

Este trabajo fue financiado por la Fundação Cecília Zino.

\section{References}

Acri, M., Hooley, C. D., Richardson, N., y Moaba, L. B. (2017). Peer models in mental health for caregivers and families. Community Mental Health Journal, 53(2), 241-249. https://doi.org/10.1007/s10597-016-0040-4

Akers, R. L. y Jennings, W. G. (2019). The Social Learning Theory of Crime and Deviance. En M. Krohn, N. Hendrix, G. Penly Hall y A. Lizotte (Eds.), Handbook on Crime and Deviance (pp. 113-129). https://doi.org/10.1007/9783-030-20779-3_6

Australian Institute of Family Studies (2015). Communities for Children Facilitating Partners Evidence-based programme profiles: Empowering Parents, Empowering Communities (EPEC). https://apps.aifs.gov.au/ cfca/guidebook/programs/empowering-parents-empowering-communities-epec

Baker, S., Sanders, M. R., Turner, K. M. T. \& Morawska, A. (2017). A randomized controlled trial evaluating a lowintensity interactive online parenting intervention, Triple P Online Brief, with parents of children with early onset conduct problems. Behaviour Research and Therapy, 91, 78-90. https://doi.org/10.1016/j. brat.2017.01.016

Barlow, J. y Coren, E. (2017). The Effectiveeness of Parenting Programs: A Review of Campbell Reviews. Research on Social Work Practice, 28(1), 99-102. https://doi.org/10.1177/1049731517725184

Bernal, G., Bonilla, J., y Bellido, C. (1995). Ecological validity and cultural sensitivity for outcome research: Issues for cultural adaptation and development of psychosocial treatments with Hispanics. Journal of Abnormal Child Psychology, 23(1), 67-82. https://doi.org/10.1007/BF01447045

Bernal, C., Jiménez-Chafey, M. I., y Rodríguez, M. M. D. (2009). Cultural adaptation of treatments: A resource for considering culture in evidence-based practice. Professional Psychology: Research and Practice, 40(4), 361-368. https://doi.org/10.1037/a0016401

Bevilacqua, L., Hale, D., Barker, E. D. y Viner, R. (2018). Conduct problems trajectories and psychosocial outcomes: a systematic review and meta-analysis. European Child \& Adolescent Psychiatry, 27(4), 1239-1260. https:// doi.org/10.1007/s00787-017-1053-4 
Cortis, N. (2012). Overlooked and under-served? Promoting service use and engagement among 'hard-toreach' populations. International Journal of Social Welfare, 21(4), 351-360. https://doi.org/10.1117/.j.14682397.2011.00825.x

Day, C. y Nicoll, J (2017) Empowering parents empowering communities: Programme theory. South London \& Maudsley NHS Foundation Trust.

Day, C., Kearney, C. y Squires, F. (2017). Art, Science and Experience of Peer Support: Learning from the Empowering Parents, Empowering Communities Programme. International Journal of Birth and Parent Education, 4(2).

Day, C., Michelson, D., Thomson, S., Penney, C. y Draper, L. (2012a). Innovations in Practice: Empowering Parents, Empowering Communities: A pilot evaluation of a peer-led parenting programme. Child and Adolescent Mental Health, 17(1), 52-57. https://doi.org/10.1111/j.1475-3588.2011.00619.x

Day, C., Michelson, D., Thomson, S., Penney, C. y Draper, L. (2012b). Evaluation of a peer led parenting intervention for disruptive behavior problems in children: community based randomized controlled trial. BMJ, 344(7849), 1-10. https://doi.org/10.1136/bmj.e1107

Day, C., Nicoll, J., Harwood, J., Kendall, N., Kearney, L. y Kirkwood, J. (2020) Transforming Chidlren's Lives: EPEC Scaling Programme. NESTA/Department of Digital Culture Media \& Sport. South London \& Maudsley NHS Foundation Trust.

Domenech-Rodríguez, M. y Wieling, E. (2004). Developing culturally appropriate, evidence-based treatments for interventions with ethnic minority populations. In M. Rastogi \& E. Wieling (Eds.), Voices of color: First-person accounts of ethnic minority therapists (pp. 313-333). Sage.

Early Intervention Foundation (2016). Guidebook: Empowering Parents Empowering Communities. http://guidebook.eif.org.uk/programme/empowering-parents-empowering-communities

Eisler, I. y Lask, J. (2015). Family Interventions. In A. Thapar, D. S. Pine, J. F. Leckman, S. Scott, M. J. Snowling y E. Taylor (Eds.), Rutter's Child and Adolescent Psychiatry (6 $6^{\text {th }}$ Edition) (pp. 510-520). John Wiley \& Sons, Ltd. https://doi.org/10.1002/9781118381953

Eyberg, S., Boggs, S., y Algina, J. (1995). Parent-child interaction therapy: A psychosocial model for the treatment of young children with conduct problem behavior and their families. Psychopharmacology Bulletin, 31(1), 83-91.

Farrington, D. P., Gaffney, H., Lösel, F.y Ttofi, M. M. (2017). Systematic reviews of the effectiveness of developmental prevention programs in reducing delinquency, aggression, and bullying. Aggression and Violent Behavior, 33, 91-106. https://doi.org/10.1016/j.avb.2016.11.003

Gardner, F., Burton, J., y Klimes, I. (2006). Randomised controlled trial of a parenting intervention in the voluntary sector for reducing child conduct problems: Outcomes and mechanisms of change. Journal of Child Psychology and Psychiatry, 47(11), 1123-1132. https://doi.org/10.1111/j.1469-7610.2006.01668.x

Harwood, J. (2017). The impact of a behavioural parenting intervention on parental reflective function [Doctoral dissertation, University of London]. EThOS. https://ethos.bl.uk/OrderDetails.do?uin=uk.bl.ethos.792695

Hoagwood, K. E., Cavaleri, M. A., Olin, S. S., Burns, B. J., Slaton, E., Gruttadaro, D., y Hughes, R. (2010). Family support in children's mental health: A review and synthesis. Clinical Child and Family Psychology Review, 13(1), 1-45. https://doi.org/10.1007/s10567-009-0060-5

Jolliffe, D., Farrington, D. P., Piquero, A. R., Loeber, R. y Hill, K. G. (2017). Systematic review of early risk factors for lifecourse-persistent, adolescence-limited, and late-onset offenders in prospective longitudinal studies. Aggression and Violent Behavior, 33, 15-23. https://doi.org/10.1016/j.avb.2017.01.009

Kaminski, J. W. y Claussem. A. H. (2017). Evidence Base Update for Psychosocial Treatments for Disruptive Behaviors in Children. Journal of Clinical Child \& Adolescent Psychology, 46(4), 477-499. https://doi.org/10.108 0/15374416.2017.1310044

Leijten, P., Gardner, F., Melendez-Torres, G. J., Jolien van Aar, J., Hutchings, J., Schulz, S., Knerr, W. y Overbeek, G. (2019). What to Teach Parents to Reduce Disruptive Child Behavior: Two Meta-Analyses of Parenting Program Components. Journal of the American Academy of Child \& Adolescent Psychiatry, 58(2), 180190. https://doi.org/ 10.1016/j.jaac.2018.07.900 
Majumder, A. (2015). The impact of parenting style on children's educational outcomes in the United States. Journal of Family and Economic Issues, 37, 1-10. https://doi.org/ 10.1007/ s10834-015-9444-5

Meija, A., Leijten, P., Lachman, J. M. y Parra-Cardona, J. R. (2017). Different Strokes for Different Folks? Contrasting Approaches to Cultural Adaptation of Parenting Interventions. Prevention Science, 18, 630-639. https://doi.org/10.1007/s11121-016-0671-2

Nystrand, C., Feldman, I., Enebrink, P. \& Sampaio, F. (2019). Cost-effectiveness analysis of parenting interventions for the prevention of behaviour problems in children. PloS ONE, 14(12). https://doi.org/10.1371/journal.pone.0225503

O'Brien, M. y Daley, D. (2011). Self-help parenting interventions for childhood behaviour disorders: a review of the evidence. Child: Care, Health, and Development, 37(5),623-637. https:// doi.org/10.1111/j.1365-2214.2011.01231.x

Pedersen, G. A., Smallegange, E., Coetzee, A., Hartog, K., Turner, J., Jordan, M. J. D. y Brown, F. L. (2019). A Systematic Review of the Evidence for Family and Parenting Interventions in Low- and Middle-Income Countries: Child and Youth Mental Health Outcomes. Journal of Child and Family Studies, 28, 2036-2055. https:// doi.org/10.1007/s10826-019-01399-4

Sanders, M. R. (1999). Triple P - Positive Parenting Program: Towards an empirically validated multilevel parenting and family support strategy for the prevention of behavior and emotional problems in children. Clinical Child and Family Psychology Review, 2(2), 71-90. https://doi.org/10.1037/0893-3200.22.3.506

Scott, S., O'Connor, T. G., Futh, A., Matias, C., Price, J., y Doolan, M. (2010). Impact of a parenting program in a high-risk, multiethnic community: The PALS trial. Journal of Child Psychology and Psychiatry, 51(12), 1331-1341. https://doi.org/10.1117/j.1469-7610.2010.02302.x

Scott, S. y Gardner, F. (2015). Parenting Programs. In A. Thapar, D. S. Pine, J. F. Leckman, S. Scott, M. J. Snowling \& E. Taylor (Eds.), Rutter's Child and Adolescent Psychiatry (6 ${ }^{\text {th }}$ Edition) (pp. 483-495). John Wiley \& Sons, Ltd. https://doi.org/10.1002/9781118381953

Thomson, S., Michelson, D. y Day, C. (2014). From parent to "peer facilitator": a qualitative study of a peer-led parenting programme. Child: Care Health and Development, 41(1), 76-83. https://doi.org/ 10.1111/cch.12132

Tully, L. A., Collins, D. A. J., Piotrowska, P. J., Mairet, K. S., Hawes, D. J., Moul, C. ... Dadds, M. R. (2018). Examining Practitioner Competencies, Organizational Support and Barriers to Engaging Fathers in Parenting Interventions. Child Psychiatry \& Human Development, 49, 109-122. https://doi.org/10.1007/s10578-017-0733-0

Tully, L. A., Piotrowska, P. J., Collins, D. A. J., Mairet, K. S., Black, N., Kimonis, E. R. ... Dadds, M. R. (2017). Optimising child outcomes from parenting interventions: fathers' experiences, preferences and barriers to participation. BMC Public Health, 17, 550-564. https://doi.org/10.1186/s12889-017-4426-1

Vasileva, M., Graf, R. K., Reinelt, T., Petermann, U.y Petermann, F. (2020). Research review: A meta-analysis of the international prevalence and comorbidity of mental disorders in children between 1 and 7 years. Journal of Child Psychology and Psychiatry, 62(4), 372-381. https://doi.org/10.1117/jcpp.13261

Wachs, T. D., Georgieff, M., Cusick, S. y McEwen, B. S. (2014). Issues in the timing of integrated early interventions: Contributions from nutrition, neuroscience, and psychological research. Annals of the New York Academy of Sciences, 1308(1), 89-106. https://doi.org/10.1111/nyas.12314

Webster-Stratton, C. (2001). The parent and child series: A comprehensive course divided into four programsLeaders'guide. The Incredible Years.

Webster-Stratton, C. y Reid, M. J. (2017). In Incredible Years Parents, Teachers, and Children Training Series: A Multifaceted Treatment Approach for Young Children with Conduct Problems. In J. R. Weisz y A. E. Kazdin, Evidence-Based Psychotherapies for Children and Adolescents (3rd Ed) (pp 122-141). The Guilford Press.

Winter, R. (2013). Empowering Parents Empowering Communities. Murdoch Children's Research Institute/Tasmanian Early Years Foundation. https://socialplanningtool.net.au/wp-content/uploads/2019/04/EPEC_Evaluation_Final.pdf

WHO (2016). INSPIRE: Seven strategies for ending violence against children. World Health Organization. https:// www.who.int/publications/i/item/inspire-seven-strategies-for-ending-violence-against-children

Yalom, I. D. y Leszcz, M. (2020). The Theory and Practice of Group Psychotherapy (6 $6^{\text {th }}$ Ed). Basic Books. 


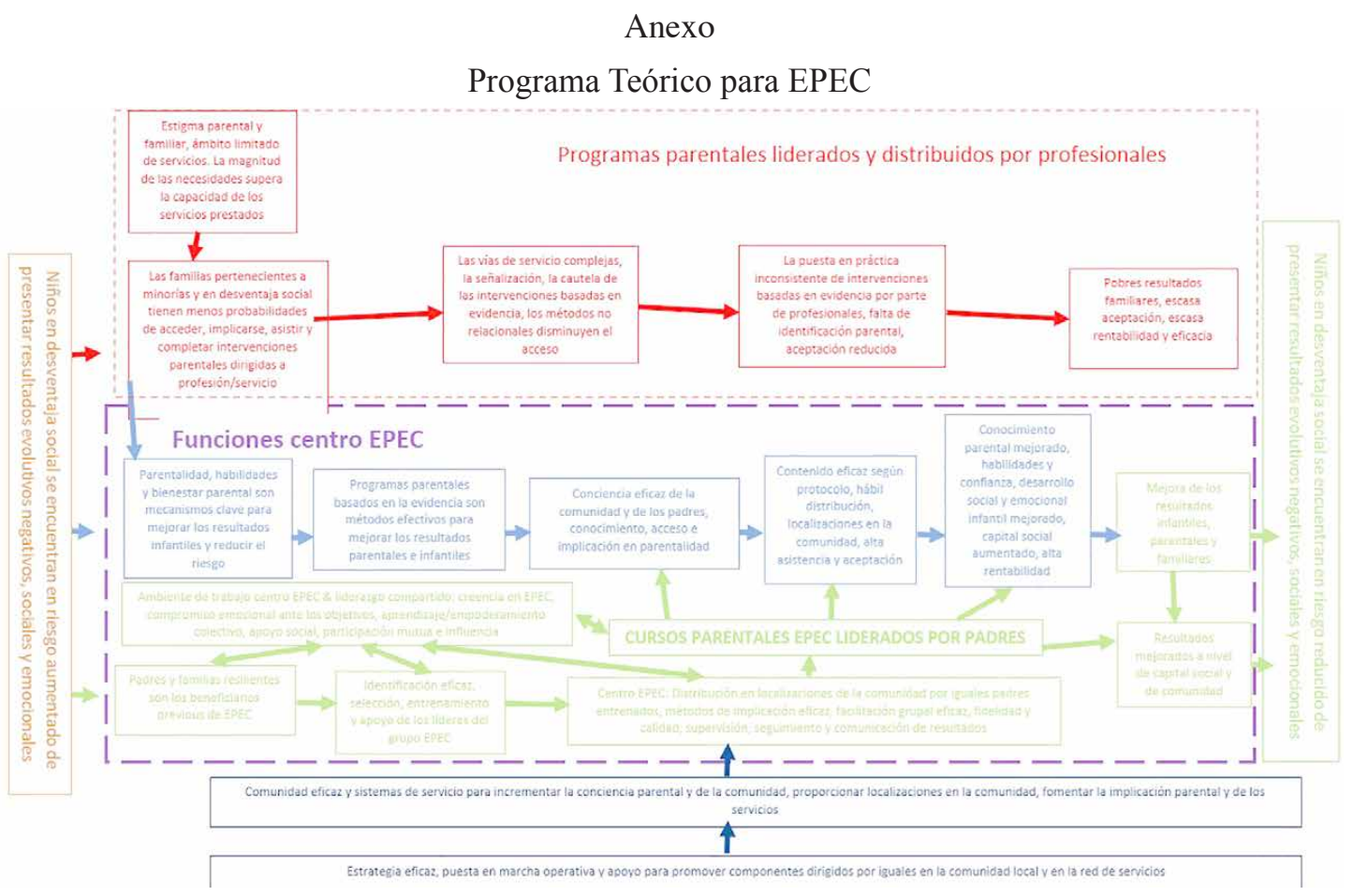

